\title{
ENFERMEDAD PERIODONTAL ASOCIADA AL EMBARAZO
}

\author{
PERIODONTAL DISEASE ASSOCIATED WITH PREGNANCY
}

\author{
Raisa Cuya García' \\ cgraysitaale@gmail.com \\ ORCID: 0000-0001-9529-669X \\ Aracelly Chávez Raymi \\ aracellymayte@gmail.com \\ ORCID: 0000-0002-4971-4683
}

\author{
Stephanie Flores Culqui ${ }^{1}$ \\ skfc89@gmail.com \\ ORCID: 0000-0002-9437-3400 \\ Miossotty Párraga Navarro ${ }^{1}$ \\ miossottyparraga@gmail.com \\ ORCID: 0000-0002-3323-7480
}

\author{
Rossana Quinto Benalcázar \\ rossana.quintob@gmail.com \\ ORCID: 0000-0001-5308-2984 \\ Óscar Tafur Vásquez \\ otafurv@gmail.com \\ ORCID: 0000-0002-7169-1895
}

\section{RESUMEN}

La gingivitis es la enfermedad más prevalente, pues afecta entre un $5 \%$ y un $70 \%$ de la población mundial, y puede incluso llegar hasta un 90\%. En cambio, la enfermedad periodontal (EP) alcanza un promedio del $30 \%$ al $80 \%$, y es más frecuente a partir del segundo trimestre de embarazo. En América Latina, afecta entre un $30 \%$ y un $40 \%$ de la población. Se estima que durante el embarazo hay un mayor riesgo a contraer enfermedad periodontal en una proporción de 1 de cada 5 mujeres. La enfermedad periodontal es una enfermedad inflamatoria que perjudica a los tejidos de soporte del diente (encía, cemento radicular, ligamento periodontal y hueso alveolar).

Se ha demostrado que existe una relación directa entre el agravamiento de la EP con el embarazo. Esto se debe a la variación hormonal que ocurre en este periodo, la cual promueve el crecimiento excesivo de microorganismos patógenos responsables de la inflamación gingival. Entre estos microorganismos patógenos encontramos a Prevotella intermedia y Porphyromonas gingivalis. Esta última, junto a Fusobacterium nucleatum, son capaces de atravesar la barrera placentaria y causar infecciones y resultados adversos en el embarazo, tales como parto prematuro, preeclampsia y muerte fetal.

Palabras clave: enfermedad periodontal, gingivitis, embarazo, microbioma oral, resultados adversos en el embarazo

\section{ABSTRACT}

Gingivitis is the most prevalent disease worldwide, affecting from 5 to $70 \%$ of the population and reaching up to $90 \%$. On the other hand, the mean prevalence of periodontal disease (PD) is 30 to $80 \%$, being more frequent in the second trimester of pregnancy. Periodontal disease is an inflammatory disease of the tissues supporting (gingiva, root cement, periodontal ligament and alveolar bone). In Latin America, PD affects between 30 and $40 \%$ of the population, and it is

Citar como: Cuya- García R, Chávez-Raymi A, Flores- Culqui S, Párraga-Navarro M, Quinto-Benalcazar R, Tafur-Vásquez O. Enfermedad periodontal asociada al embarazo. Rev Cient Odontol (Lima). 2019; 7 (1): 132-139. 
estimated that 1 to 5 women will develop PD during pregnancy. It has been shown that there is a direct relationship between the aggravation of PD and pregnancy. This is due to hormonal changes during gestation, which promote the excessive growth of pathogenic microorganisms responsible for gingival inflammation.

These pathogenic microorganisms include Prevotella intermedia and Porphyromonas gingivalis. The latter, together with Fusobacterium nucleatum. are able to cross the placental barrier causing infections and adverse pregnancy outcomes, such as premature birth, preeclampsia, fetal death and metrial arteritis.

Keywords: Periodontal disease, gingivitis, pregnancy, oral microbiome, adverse pregnancy outcomes.

\section{INTRODUCCIÓN}

La gingivitis es la enfermedad más prevalente y puede incluso llegar hasta un $90 \%$ de la población mundial. Este porcentaje varía según la zona geográfica y los parámetros. Diferente es la situación de la enfermedad periodontal (EP), que alcanza un promedio del $30 \%$ al $80 \%$, y es más frecuente a partir del segundo trimestre de embarazo $\left({ }^{3}\right)$. En América Latina, afecta entre un 30\% y $40 \%$ de la población ( ${ }^{4}$ ). Se estima que durante el embarazo hay un mayor riesgo a tener enfermedad periodontal en una proporción de 1 de cada 5 mujeres $\left({ }^{1,4}\right)$. La enfermedad periodontal es un conjunto de enfermedades inflamatorias que perjudican los tejidos de soporte del diente (encía, cemento radicular, ligamento periodontal y hueso alveolar) $\left(^{5}\right)$.

Existen factores de riesgo que pueden empeorar la salud bucodental en la embarazada. Estos pueden ser intrínsecos (hormonales, vasculares, salivales, microbiológicos e inmunológicos) o extrínsecos (acumulación de placa bacteriana, ingestión de alimentos azucarados, dieta cariogénica, disminución en la frecuencia del cepillado y deficiente nivel de conocimiento sobre salud bucal) $\left(^{6}\right)$.

Se ha demostrado que existe una relación directa entre el agravamiento de la EP y el embarazo $\left({ }^{1}\right)$. Esto se debe a la variación hormonal que ocurre en este periodo, la cual promueve el crecimiento excesivo de microorganismos patógenos responsables de la inflamación gingival $\left({ }^{7}\right)$. Entre estos microorganismos patógenos encontramos a Prevotella intermedia y Porphyromonas gingivalis. Esta última, junto con Fusobacterium nucleatum, son capaces de atravesar la barrera placentaria y causar infecciones y resultados adversos en el embarazo, tales como parto prematuro, preeclampsia, muerte fetal y arteritis metrial $(1,8)$.

\section{MECANISMO DE ACCIÓN}

Se caracteriza por la acumulación de bacterias periodontales en forma de biofilm, lo que produce irritación, inflamación y destrucción de tejido periodontal. Durante este periodo, los cambios hormonales incrementan la permeabilidad de los vasos sanguíneos y esto facilita la entrada de agentes patógenos orales ( $P$. gingivalis, F. nucleatum) y de mediadores de la inflamación en la circulación sanguínea (citoquina IL6, IL8, etc.). Una vez en el torrente sanguíneo, los derivados de los patógenos orales y los mediadores de la inflamación pueden diseminarse sistemáticamente y producir inflamación e infección de la placenta $\left({ }^{9}\right)$.

Según Tettamanti et al. (1), la EP tiende a una mayor prevalencia de enfermedades sistémicas, como enfermedades cardiovasculares, diabetes, trastorno pulmonar obstructivo crónico y ateroesclerosis. 
Los resultados adversos (APO) pueden manifestarse con una prevalencia de aproximadamente un $2 \%$ a $3 \%$, y son la principal causa de morbilidad y mortalidad materna en Occidente, por lo que la magnitud de la asociación ha variado y los estudios muestran hallazgos contradictorios. Varios estudios epidemiológicos encontraron asociación entre EP y APO en el embarazo, mientras que otros usaron diferentes métodos de medición e investigación en varias poblaciones y coinciden en que hay una posible relación entre EP y APO durante el embarazo. Por ahora, este sigue siendo un problema no resuelto $\left({ }^{1}\right)$.

\section{MICROBIOMA}

\section{ORAL}

Existe una relación directa entre el empeoramiento de la enfermedad periodontal (EP) y el embarazo, lo que ha sido demostrado en muchos estudios. En el transcurso de la gestación, las manifestaciones clásicas de la EP, tales como sangrado al sondaje y aumento de profundidad de las bolsas periodontales, se exacerban; sin embargo, estos signos clínicos se reducen después del parto. Estudios recientes encontraron que la EP no afecta a las mujeres embarazadas más que a las no embarazadas (1). Así se demostró en el estudio de Borgo et al., cuando analizaron los valores de índice de placa visible (VPI), el índice de sangrado gingival (IEG), la profundidad de sondeo (PD), el nivel de inserción clínica $(C A L)$ y el sangrado en el sondeo (POB) en mujeres embarazadas y no embarazadas, evidenciándose un incremento en la inflamación gingival en ambos grupos. Sin embargo, se observó un alza significativa en estos valores durante el segundo y tercer trimestre de embarazo, que fue más severo en este último como consecuencia de la variación hormonal propia de este periodo y que promueve un crecimiento excesivo de los microorganismos patógenos responsables de la inflamación gingival $\left({ }^{7}\right)$.

La EP es una respuesta inflamatoria del huésped a la presencia de placa dental, lo que conduce a la pérdida de dientes si no se trata. La presencia de estrógeno y progesterona en altas concentraciones contribuye al crecimiento bacteriano, la exacerbación del edema gingival y la vascularización, y las bolsas periodontales servirán como reservorio para estos microorganismos $\left({ }^{1,7}\right)$.

Las modificaciones en la microbiota oral se consideran como un mecanismo potencial para el desarrollo de la EP durante el embarazo. Un estudio reciente informó que el agravamiento de la EP se asoció con el aumento de bacterias del "complejo rojo", como Porphyromonas gingivalis y Prevotella intermedia; sin embargo, en otro estudio reciente, se informó que la carga de bacterias de Porphyromonas gingivalis y Tannerella forsythia empeoran la EP en el tercer mes de embarazo al presentar sangrado en el sondeo gingival. Se ha demostrado que las bacterias, y en particular Fusobacterium nucleatum, que se originan en la bolsa periodontal de las mujeres embarazadas, atraviesan la barrera placentaria y causan infecciones agudas, así como resultados adversos en el embarazo $\left(^{1}\right)$.

\section{PLACENTARIA}

Hace mucho tiempo, se creía que la placenta era estéril en la gestación normal, pero varios autores han señalado que la placenta en embarazos saludables tiene su propio microbioma residente. Se ha demostrado que no existe una asociación entre parto prematuro y una invasión intraamniótica relativamente benigna de bacterias, pero sí cuando hay presencia de una combinación de invasión bacteriana y respuesta inflamatoria concomitante. Los microbios 
causantes de la infección bacteriana, en un $50 \%$ de las pacientes, eran de un género y una especie que se encuentra comúnmente en la cavidad oral: Fusobacterium nucleatum y Porphyromonas gingivalis. Esta última tiene la capacidad de interactuar con microbios normalmente benignos y convertirlos en patógenos activos que desempeñan un papel siniestro en la disbiosis microbiana, lo que conduce a resultados adversos del embarazo como parto prematuro, preeclampsia y muerte fetal. Por eso, el perfil taxonómico bacteriano del microbioma de la placenta es más similar al de la cavidad oral que a las floras de la piel o los tejidos nasales, vaginales o gastrointestinales $\left({ }^{10}\right)$.

El éxito de un embarazo depende de la correcta remodelación de las arterias espirales uterinas, cuyo fin es que la placenta reciba un suministro de sangre adecuado sin flujo turbulento pulsátil. Porphyromonas gingivalis es una bacteria gramnegativa, anaeróbica, capaz de interferir con la remodelación de las arterias uterinas en espiral e inducir a una arteritis metrial $\left(^{8}\right)$.

\section{VAGINAL}

El Proyecto Microbioma Humano (2012) informó que el microbioma vaginal muestra una diversidad microbiana sin complicaciones, en comparación con la del tracto gastrointestinal o la cavidad oral. En sujetos sanos, parece haber una variación mínima en la diversidad de la microbiota vaginal. Las especies de lactobacilos dominan típicamente la microbiota vaginal, que comprende más del $70 \%$ de la microflora. Este predominio, generalmente, evita la colonización por parte de especies extrínsecas indeseables y, por lo tanto, puede proporcionar una función protectora. El estudio de Prince et al. señala que, durante el embarazo, se produce un aumento general de bacterias de los taxones de lactobacilos, clostridiales, bacteroides y actinomycetales. Sin embargo, ninguno de estos taxones mostró tener efectos negativos en el embarazo. Hyman et al. encontraron que los cambios en las proporciones de lactobacilos en el microbioma vaginal y la presencia de bacterias nocivas no se correlacionaron con el parto prematuro $\left({ }^{8,10}\right)$.

\section{RESPUESTA DEL HUÉSPED}

En el embarazo, la respuesta inmune juega un papel fundamental para el mantenimiento de un equilibrio saludable entre la madre y el feto.

La destrucción del tejido de soporte periodontal es producida por el desbalance de la respuesta inmune generada por el paciente frente a antígenos y factores de virulencia derivados de patógenos periodontales $\left({ }^{11}\right)$.

La enfermedad periodontal es considerada un factor de riesgo en nacimientos prematuros con bajo peso, con graves lesiones e incluso la muerte en los primeros meses de vida, causada principalmente por bacterias anaerobias gramnegativas productoras de liposacáridos, la cuales disminuyen la capacidad de respuesta inmune celular $\left({ }^{12}\right)$.

Las bacterias o endotoxinas bacterianas en la circulación sistémica pueden inducir a la producción de citoquinas, las cuales activan aún más la respuesta inflamatoria de las moléculas que involucran IL-6 y la proteína $\mathrm{C}$ reactiva. La respuesta inflamatoria también activa las células inflamatorias y endoteliales, y puede resultar en disfunción endotelial $\left({ }^{1}\right)$.

El nivel reducido de IL-1b e IFN-gamma salival observado en mujeres embarazadas puede atribuirse a los efectos hormonales de las citoquinas. Los niveles 
salivales reducidos de IFN gamma pueden explicarse por los informes de que una de las principales alteraciones en el sistema inmunológico durante el embarazo es la disminución parcial de las respuestas inmunitarias $\left({ }^{13}\right)$.

\section{RESULTADOS ADVERSOS}

Está demostrado que los nacimientos prematuros pueden ser causados por la infección vaginal e intrauterina, y se relacionan en un $50 \%$ con infecciones intraamnióticas, lo cual tiene como consecuencia que el niño nazca antes de tiempo o se produzca una preeclampsia $\left({ }^{10}\right)$.

\section{TRATAMIENTO}

Debido a los constantes cambios hormonales y fisiológicos que ocurren en el embarazo, como el aumento del riego sanguíneo, se produce una marcada vasodilatación en las encías, y la falta de una correcta higiene oral trae como consecuencias una mayor acumulación de placa bacteriana y sangrado de las encías en la gestante, lo que resulta bastante molesto.

El objetivo del tratamiento periodontal durante la gestación es minimizar la potencial respuesta inflamatoria exagerada a la placa bacteriana y evitar que se llegue a tratamientos que requieran intervención quirúrgica $\left({ }^{14}\right)$.

Pero ¿en qué momento se debería realizar el tratamiento periodontal? El periodo más adecuado para llevar a cabo cualquier abordaje dental es el segundo trimestre del embarazo, con mayor razón si vamos a realizar un procedimiento quirúrgico.

La forma más común de tratar la gingivitis es desbridando o eliminando la placa dental o cálculo de los dientes mediante el ultrasonido; reduciendo los factores de riesgo, tales como el cambio de una dieta llena de carbohidratos por una dieta rica en frutas, vegetales y proteínas; así como una limpieza profesional cada 6 meses. Mientras que la periodontitis se trata con desbridamiento y otros medios mecánicos que pueden implicar cirugía.

Una vez que el aspecto clínico y la pérdida ósea son evidentes, lo que se buscará en el tratamiento será controlar la inflamación, detener la progresión de la enfermedad y crear las condiciones que ayuden a la paciente a mantener una dentición sana, funcional y cómoda a largo plazo $\left({ }^{15}\right)$.

El tratamiento puede ser dividido en diferentes fases, de acuerdo con las medidas que se realizan:

a. Fase I. Terapia no quirúrgica. Es la piedra angular de la terapia periodontal y el primer enfoque recomendado para el control de las infecciones periodontales. Se le conoce también como "terapia relacionada con la causa", "terapia de fase I o fase etiotrófica" y "terapia inicial" ${ }^{(16)}$. La terapia periodontal no quirúrgica comienza motivando e instruyendo al paciente sobre el cuidado personal adecuado, seguido de una reevaluación de su nivel de higiene oral $\left({ }^{17}\right)$.

Se define como "eliminación de placa, control de placa, raspaje supragingival y subgingival de la raíz (SRP), y adyuvante de uso de agentes químicos". En el estudio de Winkel et al. se encontró que la combinación de metronidazol y amoxicilina produce más reducción de la profundidad de las bolsas periodontales que la medicación de control. Los antibióticos más comúnmente usados para los organismos periodontales son metronidazol, amoxicilina, tetraciclina, clindamicina, azitromicina, ciprofloxacina y augmentina $\left({ }^{16}\right)$. 
b. Fase II. Correctora. Se le conoce también como "fase quirúrgica". Incluye medidas terapéuticas tradicionales, como cirugía periodontal, terapia periodontal, tratamiento restaurador y protésico $\left({ }^{15}\right)$. Cuando no se puede eliminar el cálculo dental mediante el alisado radicular convencional, se debe realizar un colgajo abierto de la encía con la finalidad de proporcionar mayor visibilidad y acceso a la lesión $\left({ }^{16}\right)$.

La cirugía de reducción de bolsa periodontal incluye la resección de tejido blando y duro con diversas técnicas. La cirugía regenerativa se refiere a la regeneración tisular guiada usando membranas de barrera para dirigir el crecimiento de nuevo periodonto, evitando que el epitelio y el tejido conjuntivo crezcan en áreas donde se desea el crecimiento del ligamento óseo y periodontal. Se ha introducido un nuevo procedimiento con láser como una alternativa conservadora a la terapia quirúrgica. El nuevo procedimiento de inserción asistido por láser (LANAP) utiliza un láser Nd: YAG, en lugar de un bisturí y suturas, para la despielitización inicial de la bolsa periodontal y la coagulación final de la fibrina, y no incluye una elevación extensa del colgajo gingival $\left({ }^{16,17}\right)$.

c. Fase III. De mantenimiento. Consiste en la prevención de la recidiva de la enfermedad, por lo que es necesario e importante que a cada paciente se le diseñe un sistema de visitas periódicas que incluyan un programa de control de la placa (controlado profesionalmente), medidas de tartrectomía, alisado radicular y aplicación de flúor $\left({ }^{14}\right)$.

\section{CONCLUSIONES}

Es necesario concientizar a la paciente gestante acerca de la importancia de la consulta con el odontopediatra para que tenga cuidado sobre su estado de salud oral, antes, durante y después del nacimiento de su niño, ya que puede repercutir en efectos adversos no deseados, tales como nacimiento prematuro.

Es preciso mantener una excelente higiene oral cepillándose 3 veces al día y utilizando hilo dental. Puede usar colutorios a partir del séptimo mes, una vez al día, por un minuto, con clorhexidina al $0,12 \%$.

Debe consumir pocos dulces o caramelos y chicles con altos contenidos de azúcar, o al menos consumir chicles sin azúcar que contengan xilitol, y tener una dieta rica en vitaminas $C, D, A$ y calcio, que ayudan a la mineralización de huesos y dientes.

Contribución del autor: Raisa Cuya García, Aracelly Chávez Raymi, Stephanie Flores Culqui, Miosotty Párraga Navarro, Rossana Quinto-Benalcázar, Oscar Tafur Vásquez han participado en la concepción del artículo, la recolección de información, su redacción y aprobación de la versión final.

Fuente de financiamiento: Autofinanciado.

Conflicto de interés: Los autores declaran no tener conflicto de interés de ningún tipo. 


\section{REFERENCIAS BIBLIOGRÁFICAS}

1. Tettamanti L, Lauritano D, Nardone M, Gargari M. Pregnancy and periodontal disease: does exist a two-way relationship? Oral Implantol. 2017; 10 (2): 112-8. doi:10.1111/jog.13782.

2. Romanelli H. A propósito del Día de la Salud de las Encías: la salud periodontal es posible. Rev Asoc Odontol Argent. 2018; 106: 1-4.

3. García Morales G, Vega S, Tolentino A. Prevalencia de enfermedad periodontal en embarazadas de una unidad de medicina familiar de Acapulco, Guerrero. Aten Fam. 2016; 23 (3): 75-9.

4. Duque A. Prevalencia de periodontitis crónica en Iberoamérica. Rev Clin Periodoncia Implantol Rehabil Oral. 2016; 9 (2): 208-15. doi.org/10.1016/j. piro.2016.07.005

5. Reyes E. Periodontal disease related to mode of delivery in pregnant women at Hospital Eleazar Guzmán Barrón. In Crescendo. Ciencias de la Salud. 2015; 2 (1): 377-85.

6. Rodríguez A, León M, Arada A, Martínez M. Factores de riesgo y enfermedades bucales en gestantes. Rev Cien Méd. 2013; 17 (5): 51-63.

7. Borgo P, Rodrigues V, Feitosa A, Xavier K, Avila-Campos M. Association between periodontal condition and subgingival microbiota in women during pregnancy: a longitudinal study. J Appl Oral Sci. 2014; 22 (6): 528-33 doi: 10.1590/1678775720140164

8. Reyes L, Phillips P, Wolfe B, Golos T, Walkenhorst M, Progulske-Fox A. Porphyromonas gingivalis and adverse pregnancy outcome. J Oral Microbiol. 2018; 10 (1): 1374153. doi: 10.1080/20002297.2017.1374153.

9. Komine-Aizawa $S$, Aizawa $S$, Hayakawa S. Periodontal diseases and adverse pregnancy outcomes J. Obstet. Gynaecol. Res. 2018 doi: 10.1111/jog.13782

10. Cobb C, Kelly P, Williams K, Babbar S, Angolkar M, Derman R. Oral microbiome and adverse pregnancy outcomes. International Journal of Women's Health 2017; 9: 551-9. doi: 10.2147/IJWH.S142730

11. Michea M, Briceño C, Alcota M, González F. Péptidos antimicrobianos y mediadores lipídicos: rol en las enfermedades periodontales. Rev Clin Periodoncia Implantol Rehabil Oral. 2016; 9 (3): 231-7. doi: 10.1016/j.piro.2016.0303

12. Quispe D. Cambios hormonales y periodontales en el embarazo. Rev Actual Clín. 2013; (31): 1552-6.

13. Lasisi TJ, Abdus Salam RA. Pregnancy-induced periodontal inflammation: Influence of salivary cytokines and antimicrobial proteins, Saudi Dental Journal. 2018; 30: 306-11. doi:10.1111/j.1600-0765.2008.01119

14. Díaz-Guzmán LM, Castellanos-Suárez JL. Lesions of the oral mucosa and periodontal disease behavior in pregnant patients. Med Oral Patol Oral Cir Bucal. 2004; 9: 430-7. 
15. Kinane D, Stathopoulou P, Papapanou P. Periodontal diseases. Nat Rev Dis Primers. 2017; (17): 1-14.

16. Tanwar J, Hungund S, Dodani K. Nonsurgical periodontal therapy: A review. J Oral Res Rev 2016; 8: 39-44.

17. Mathews D. Prevention and Treatment of Periodontal Diseases in Primary Care. Evidence-Based Dentistry. 2014 Sep;15 (3): 68-9.

ARTí́culo RECIBIDO: 12-02-2019

Artículo aceptado: 13-05-2019 